\title{
Acoustic Emission Monitoring System for Hard Polishing of Sapphire Wafer
}

\author{
Chun-Wei Liu, Hong-Chang Chen, and Shih-Chieh Lin* \\ Department of Power Mechanical Engineering, National Tsing Hua University, \\ No. 101, Section 2, Kuang-Fu Road, Hsinchu 30013, Taiwan 802
}

(Received February 28, 2019; accepted May 30, 2019)

Keywords: sapphire wafer, hard polishing, acoustic emission

In this study, an acoustic emission (AE) sensor is used to monitor the hard polishing process of a sapphire wafer. Since the AE sensor is very sensitive, a specially designed polishing head is used such that the AE sensor can be placed as close to the polishing area as possible. Then, hard polishing experiments are conducted to investigate the effects of hard polishing processing factors including pressure and rotational speed on the material removal rate (MRR) and root mean square (RMS) value of AE signals. According to the results, it is shown that the RMS value of AE signals strongly correlates with the MRR of the processes under various polishing conditions. On the basis of the experimental results, a mathematical model of MRR is developed. The coefficient of determination $\left(R^{2}\right)$ of the model is about $99 \%$, the corrected coefficient of determination $\left(R_{c o r}^{2}\right)$ is about $95 \%$, and the prediction error is less than $12 \%$ in all cases studied. The results indicate that the AE sensor is a potential tool for monitoring the polishing process.

\section{Introduction}

Sapphire wafers are hard, brittle, and chemically stable; hence, polishing them is quite difficult. A series of processing steps are frequently adopted to process them such that the processing time can be reduced and the wafer quality can also be improved. Hard polishing is a process usually performed between lapping and chemical mechanical polishing (CMP). The processing time of the following CMP process can be markedly reduced by achieving an atomic level roughness of $\sim 10 \mathrm{~nm}$ and a high material removal rate (MRR).

As a nondestructive testing technology, ${ }^{(1,2)}$ acoustic emission (AE) was widely used in the monitoring of mechanical processing. ${ }^{(3-5)}$ Chang et al. ${ }^{(6)}$ used an AE sensor to monitor the MRR during the polishing process. Tang et al. ${ }^{(7)}$ classified the polishing process into several stages by analyzing AE signals in order to avoid microscratching. Hase et al. ${ }^{(8)}$ studied the characteristics of AE signals and correlated them with wear mechanisms. Aguiar et al. ${ }^{(9)}$ used neural networks to predict the surface roughness of ground workpieces on the basis of the analysis of output variables such as AE signals and cutting power. Li et al. ${ }^{(10)}$ established 
a simplified fracture propagation model of low-carbon nitrogen-enhanced (316LN) stainless steel. Besides, they also analyzed the inner connection between the energy release rate of the AE source and the morphological aspect of crack formation. Lee et al. ${ }^{(11)}$ used a microphone to collect AE signals in wet milling. The stability variation of milling dynamics could be observed using the peak frequency variation of the microphone signals.

In this work, we study the feasibility of using AE signals to monitor the hard polishing process of a sapphire wafer. Since the AE sensor is very sensitive, a polishing head is specially designed such that the $\mathrm{AE}$ sensor can be placed as close to the polishing area as possible. The design and other experimental setup are detailed in the following section. A series of experiments are then conducted to study the effects of processing factors on the MRR of the sapphire wafer during hard polishing. Furthermore, the correlations between the MRR of the process and AE signal values under different polishing conditions are studied. Then, an MRR model for the hard polishing of sapphire wafers is established. On the basis of these results, conclusions are made at the end.

\section{Experimental Setup}

Figure 1 shows the experimental setup used to monitor the hard polishing process. As shown in the figure, a carrier is designed such that the AE sensor is placed very close to the workpiece; a slip ring is used to prevent the winding of a signal wire; a sponge is used to reduce the vibration transmitted from the drive to the carrier and sensor. Three workpieces of 4 inch sapphire wafers are mounted on the polishing plate, which is composed of copper and resin. The slurry consists of ethylene glycol and diamond abrasives with a mean grain diameter of 3 $\mu \mathrm{m}$.

Preliminary tests were conducted to ensure that the devices were properly set. Figure 2 shows some of the test results; it demonstrates the effects of different polishing conditions on the AE signals. In one of these tests, the edge crack phenomenon in the sapphire wafer was found as shown in Fig. 3. The edge crack appeared when the upper holder failed to tap the polishing plate. Simultaneously, AE signals also reached a peak value, which indicated the correlation between the crack failure and the peak value of AE signals during the hard polishing and in situ processes.

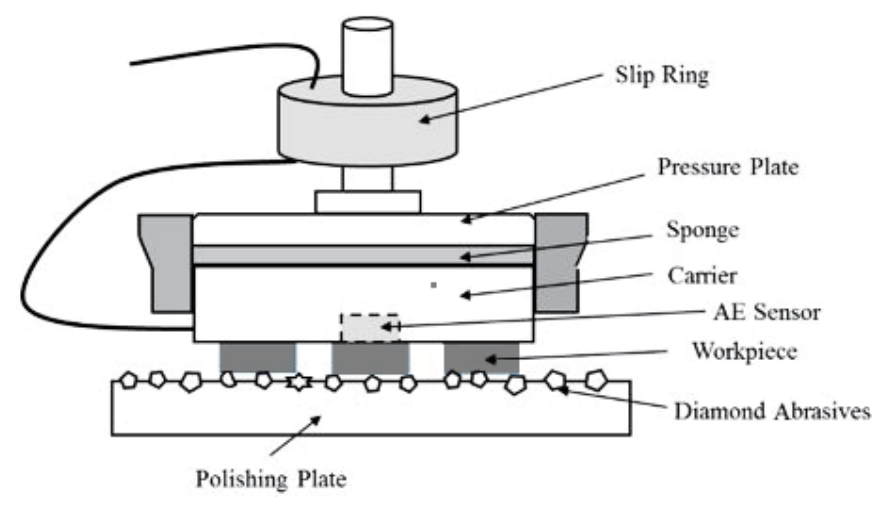

Fig. 1. Experimental setup for using AE devices to monitor the hard polishing process. 


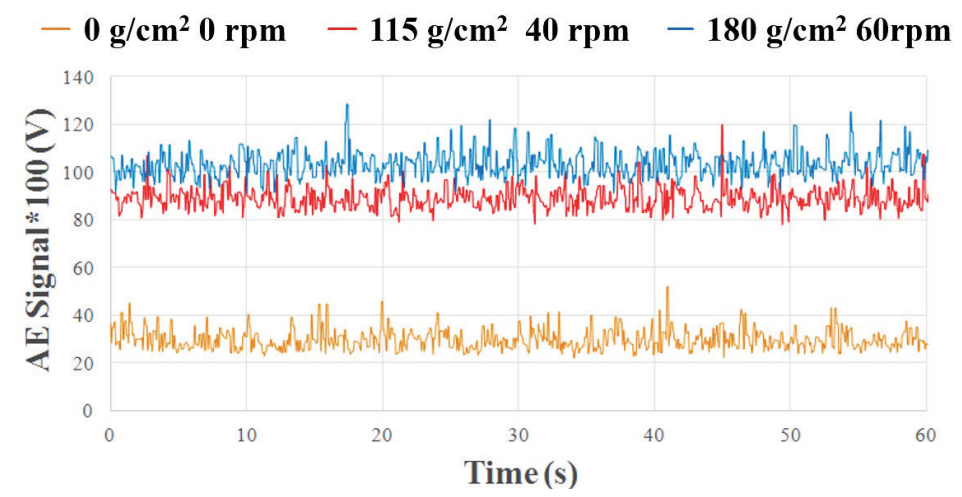

Fig. 2. (Color online) AE signals collected during hard polishing process under various conditions.

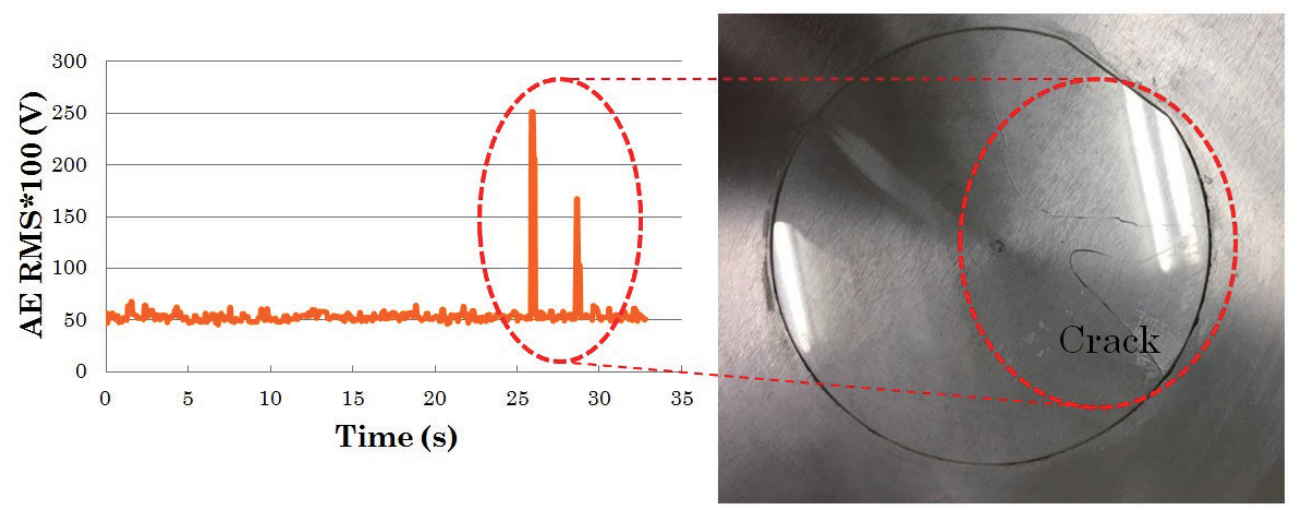

Fig. 3. (Color online) Edge crack phenomenon in sapphire wafer.

\section{Experimental Design and Results}

A series of experiments were conducted to investigate the effects of hard polishing processing factors on the MRR and root mean square (RMS) value of AE signals. The parameters studied are pressure $(P)$ and rotational speed $(V)$ at levels listed in Table 1.

For the estimation of MRR, the wafer thickness at three different locations on each sapphire wafer is measured before and after the polishing process. The average difference in the thickness before and after the polishing process at these locations is denoted as Ave. MRR. The maximum difference in the thickness before and after the polishing process among these three locations is denoted as Max. MRR.

The results of the analysis of variance (ANOVA) for the Ave. MRR, Max. MRR, and AE signals of the process are summarized in Tables 2, 3, and 4, respectively, which show that the $\mathrm{P}$ values of pressure and rotational speed are less than 0.02 in all cases studied, which will contribute to significant effects. Usually, whether the effects were significant or not depended 
Table 1

Experimental parameters and levels.

\begin{tabular}{lc}
\hline Parameters & Levels \\
\hline$P\left(\mathrm{~g} / \mathrm{cm}^{2}\right)$ & $85,115,145,180$ \\
$V(\mathrm{rpm})$ & $40,50,60$ \\
\hline
\end{tabular}

Table 2

ANOVA for Ave. MRR.

\begin{tabular}{lcccccc}
\hline Source of variation & $S S$ & DOF & MS & $F$ & $P$ & $F_{\text {critical }}$ \\
\hline$V$ & 18.430067 & 2 & 9.215033 & 38.93859 & 0.000366 & 5.143253 \\
$P$ & 12.505767 & 3 & 4.168589 & 17.61458 & 0.002228 & 4.757063 \\
Interaction & 1.4199333 & 6 & 0.236656 & - & - & - \\
Total & 32.355767 & 11 & - & - & - & - \\
\hline
\end{tabular}

Table 3

ANOVA for Max. MRR.

\begin{tabular}{lcccccc}
\hline Source of variation & $S S$ & DOF & MS & $F$ & $P$ & $F_{\text {critical }}$ \\
\hline$V$ & 9.2096167 & 2 & 4.604808 & 8.63514 & 0.017142 & 5.143253 \\
$P$ & 15.195267 & 3 & 5.065089 & 9.498278 & 0.010733 & 4.757063 \\
Interaction & 3.1995833 & 6 & 0.533264 & - & - & - \\
Total & 27.604467 & 11 & - & - & - & - \\
\hline
\end{tabular}

Table 4

ANOVA for AE signals.

\begin{tabular}{lcccccc}
\hline Source of variation & $S S$ & DOF & MS & $F$ & $P$ & $F_{\text {critical }}$ \\
\hline$V$ & 40.81155 & 2 & 20.40578 & 21.19769 & 0.001906 & 5.143253 \\
$P$ & 65.6886 & 3 & 21.8962 & 22.74595 & 0.001119 & 4.757063 \\
Interaction & 5.77585 & 6 & 0.962642 & - & - & - \\
Total & 112.276 & 11 & - & - & - & - \\
\hline
\end{tabular}

on the comparison with the experimental error. Besides, interaction effects were larger or equivalent to the experimental error theoretically. Therefore, if $P$ values showed a significant effect in the comparison with interaction effects, it would also show a significant effect in the comparison with the experimental error. In this paper, the pressure and rotational speed both show that their effects on the MRR and AE signal of the process are significant. These analysis results indicate that both the pressure and the rotational speed are critical factors that affect the MRR.

The interaction effects of pressure and speed on Ave. MRR, Max. MRR, and AE signals are shown in Figs. 4, 5, and 6, respectively. By comparing these figures, we found a similar trend, that is; the MRR and AE signal value increased with the pressure and rotational speed. This indicated that the correlation between the AE signal and the MRR could be strong. If so, the AE signals may be used as a possible tool to monitor the MRR of the process. 


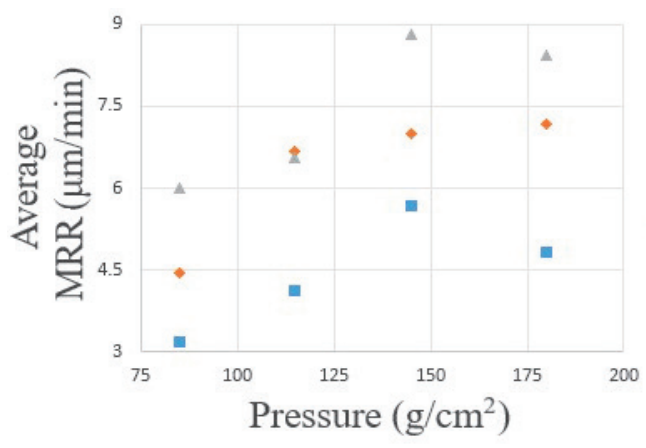

Fig. 4. (Color online) Ave. MRRs under different pressures and rotational speeds.

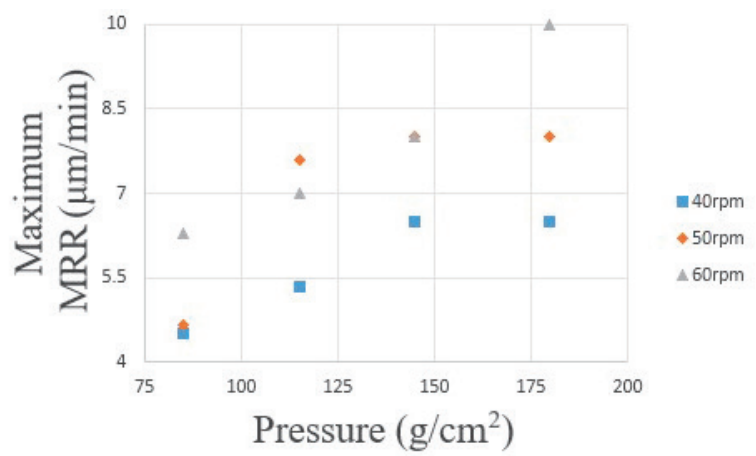

Fig. 5. (Color online) Max. MRRs under different pressures and rotational speeds.

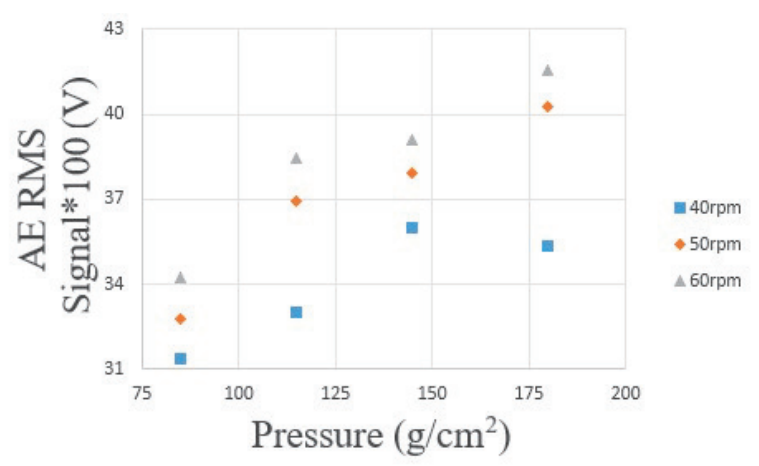

Fig. 6. (Color online) Values of AE signals under different pressures and rotational speeds.

\section{Models for MRR}

The Preston equation ${ }^{(12)}$ is a frequently adopted MRR model for the polishing process. The Preston equation is given as

$$
\mathrm{MRR}=K \times P \times V,
$$

where $K$ is the Preston coefficient reflecting the effects of all other factors such as work material, slurry, and abrasives; $P$ is the pressure per unit area; and $V$ is the relative linear velocity between the polishing plate and the workpiece.

In this study, instead of the relative linear velocity, the rotational speed was used to form the MRR model. On the basis of the collected data, the Preston equation for the MRR is derived as

$$
\begin{gathered}
\text { Ave. } \operatorname{MRR}=9.74 \times 10^{-4} \times P \times V, \\
R^{2}=97.4 \%, R_{\text {cor }}^{2}=87.1 \%,
\end{gathered}
$$




$$
\begin{gathered}
\text { Max. } \operatorname{MRR}=1.09 \times 10^{-3} \times P \times V, \\
R^{2}=98 \%, R_{\text {cor }}^{2}=88.4 \%,
\end{gathered}
$$

where $R^{2}$ and $R_{c o r}^{2}$ are the coefficient of determination and the corrected coefficient of determination, respectively, which are adopted as the measures for the fitness of the model.

$$
\begin{gathered}
R^{2}=\frac{S S(\text { model })}{S S(\text { total })} \\
R_{\text {cor }}^{2}=\frac{S S(\text { model })-S S(\text { mean })}{S S(\text { total })-S S(\text { mean })}
\end{gathered}
$$

Here, SS(model) is the sum of squares of the model, SS(total) is the sum of squares of the model and the sum of squares of the residuals, and $S S$ (mean) is the sum of squares of the mean value. In general, the higher the coefficient of determination and the corrected coefficient of determination, the better the model for estimation.

Many researchers ${ }^{(13-15)}$ used the modified Preston equation for the MRR. The Preston equation is modified by introducing exponential coefficients as

$$
\operatorname{MRR}=K \times\left(P^{n}\right) \times\left(V^{m}\right)
$$

In the case studied, the modified Preston equation for the MRR is

$$
\begin{gathered}
\text { Ave. } \operatorname{MRR}=2.51 \times 10^{-3} \times\left(P^{0.44}\right) \times\left(V^{1.45}\right) \\
R^{2}=98.9 \%, R_{\text {cor }}^{2}=91.5 \%, \\
\text { Max. MRR }=4.62 \times 10^{-2} \times\left(P^{0.31}\right) \times\left(V^{0.9}\right) \\
R^{2}=98.8 \%, R_{\text {cor }}^{2}=88.6 \% .
\end{gathered}
$$

From Figs. 4, 5, and 6, a similar trend can be found, that is, the MRR and AE signal increased as the rotational speed increased, aside from that the effects of pressure on the MRR and AE signal are also similar. It was found that with the increase in MRR, the AE signals are also increased.

Therefore, linear regression models are adopted for the MRR model:

$$
\begin{gathered}
\text { Ave. } \mathrm{MRR}=0.494 \mathrm{AE}-11.916 \\
R^{2}=99 \%, R_{\text {cor }}^{2}=84.6 \%,
\end{gathered}
$$




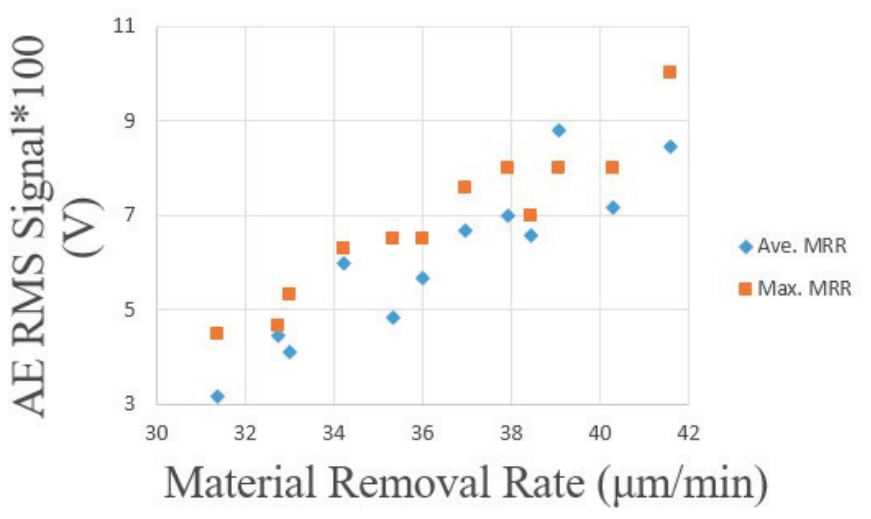

Fig. 7. (Color online) Relationship between MRR and AE RMS signals.

$$
\begin{gathered}
\text { Max. } \mathrm{MRR}=0.472 \mathrm{AE}-10.326 \\
R^{2}=99.3 \%, R_{\text {cor }}^{2}=90.3 \%
\end{gathered}
$$

where AE is the RMS value of AE signals.

If both the polishing parameters and the AE signals are used to model the MRR, the following results can be derived:

$$
\begin{gathered}
\text { Ave. MRR }=1.41 \times 10^{-4} \times\left(P^{0.15}\right) \times\left(V^{0.75}\right) \times\left(A E^{1.95}\right) \\
R^{2}=99.2 \%, R_{c o r}^{2}=93 \%, \\
\text { Max. MRR }=1.02 \times 10^{-3} \times\left(P^{-0.1}\right) \times\left(V^{-0.12}\right) \times\left(A E^{2.73}\right) \\
R^{2}=99.6 \%, R_{c o r}^{2}=95 \% .
\end{gathered}
$$

The $R^{2}$ value, $R_{c o r}^{2}$ value, average difference between prediction and experimental data, and the maximum difference between prediction and experimental data of different average removal rate models and those of different maximum removal rate models are summarized in Tables 5 and 6 , respectively. The applications of these models can be twofold: one is to predict the performance before the process; the other is the online monitoring of the process with sensor signals collected online during the process. For removal rate prediction, the modified Preston equation with AE signals is better than the Preston equation for both average and maximum MRR predictions. For online MRR monitoring, the modified Preston equation with AE signals is superior to others for the estimation of both the average and maximum MRRs in all measures. 
Table 5

Comparison of average removal rate models.

\begin{tabular}{lcccc}
\hline Model type & \multicolumn{1}{c}{$R^{2}$} & $R_{c o r}^{2}$ & Ave. difference & Max. difference \\
\hline Preston equation & $97.4 \%$ & $87.1 \%$ & $11.4 \%$ & $31.1 \%$ \\
Modified Preston equation & $98.9 \%$ & $91.5 \%$ & $10.2 \%$ & $18.9 \%$ \\
Linear regression with AE & $99 \%$ & $84.6 \%$ & $8.8 \%$ & $20.2 \%$ \\
Modified Preston equation with AE & $99.2 \%$ & $93 \%$ & $7.6 \%$ & $13.5 \%$ \\
\hline
\end{tabular}

Table 6

Comparison of maximum removal rate models.

\begin{tabular}{lcccc}
\hline Model type & \multicolumn{1}{c}{$R^{2}$} & $R_{c o r}^{2}$ & Ave. difference & Max. difference \\
\hline Preston equation & $98 \%$ & $88.4 \%$ & $11.8 \%$ & $21.9 \%$ \\
Modified Preston equation & $98.8 \%$ & $88.6 \%$ & $9.6 \%$ & $24.8 \%$ \\
Linear regression with AE & $99.3 \%$ & $90.3 \%$ & $6.8 \%$ & $16.5 \%$ \\
Modified Preston equation with AE & $99.6 \%$ & $95 \%$ & $5.3 \%$ & $10.4 \%$ \\
\hline
\end{tabular}

\section{Conclusions}

A novel approach of introducing AE signal terms into the modified Preston equation to estimate MRR is established. Overall, this study shows that the AE monitoring system is important as a guide for optimizing hard polishing process parameters and improving polishing efficiency. In this study, the effects of polishing parameters on the MRR and collected AE signals were studied first. The results show that the pressure and rotational speed have significant effects on both the MRR and AE signals. The larger the pressure and the higher the rotational speed, the larger the MRR and AE signals. Several approaches are then utilized to establish the MRR model. As a consequence, for the average removal rate, the modified Preston equation with AE signals has the lowest average and maximum errors. Likewise, for the maximum MRR, the modified Preston equation with AE signals also has the lowest average error and maximum error. The $R^{2}$ value of the prediction model is about $99 \%$, the $R_{c o r}^{2}$ value of the prediction model is about $95 \%$, and the error between the predictive value and the experimental value is almost less than $12 \%$ in all cases studied.

\section{References}

1 N. Tandon and A. Choudhury: Tribol. Int. 32 (1999) 469. https://doi.org/10.1016/S0301-679X(99)00077-8

2 A. M. Al-Ghamd and D. Mba: Mech. Syst. Sig. Process. 20 (2006) 1537. https://doi.org/10.1016/ j.ymssp.2004.10.013

3 J. Zhao, K. Wang, and Y. Guo: ICCET 6 (2010) 300. https://doi.org/10.1109/ICCET.2010.5486240

4 Q. Ren, L. Baron, M. Balazinski, and K. Jemieliniak: NAFIPS (2010) 1. https://doi.org/10.1109/ NAFIPS.2010.5548197

5 L. Gao, F. Zai, S. Su, H. Wang, P. Chen, and L. Liu: Sensors 11 (2011) 600. https://doi.org/10.3390/s110100599

6 Y. P. Chang, M. Hashimura, and D. A. Dornfeld: CIRP Ann. 45 (1996) 331. https://doi.org/10.1016/S00078506(07)63074-1

7 J. Tang, D. Dornfeld, S. K. Pangrle, and A. Dangca: J. Electron. Mater. 27 (1998) 1099. https://doi.org/10.1007/ s11664-998-0144-x 
8 A. Hase, H. Mishina, and M. Wada: Wear 292 (2012) 144. https://doi.org/10.1016/j.wear.2012.05.019

9 P. R. Aguiar, C. H. R. Martins, M. Marchi, and E. C. Bianchi: Data Acquisition Applications (INTECH Publishing, 2012) p. 298. https://doi.org/10.5772/48557

10 X. Li, Y. Shao, Y. Yu, Y. Zhang, and R. Shen: Sens. Mater. 29 (2017) 1305. https://doi.org/10.18494/ SAM.2017.1604

11 R. M. Lee, P. T. Liu, and C. C. Wang: Sens. Mater. 30 (2018) 2419.

12 F. Preston: J. Soc. Glass Tech. 11 (1927) 214.

13 Q. Luo, S. Ramarajan, and S. V. Babu: Thin Solid Films 335 (1998) 160. https://doi.org/10.1016/S00406090(98)00896-7

14 W. T. Tseng, J. H. Chin, and L. C. Kang: J. Electrochem. Soc. 146 (1999) 4273. https://doi.org/10.1149/1.1392627

15 C. C. Wang, S. C. Lin, and H. Hochen: Int. J. Mach. Tools Manuf. 42 (2002) 979. https://doi.org/10.1016/S08906955(02)00004-4

\section{About the Authors}

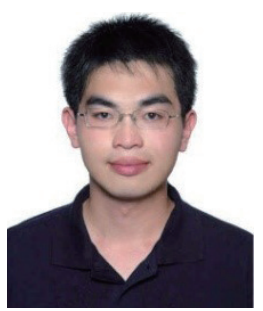

Chun-Wei Liu received his Ph.D. degree in Department of Power Mechanical Engineering from National Tsing Hua University, Taiwan, in 2015. From 2016 to 2019, he was a researcher at the Industrial Technology Research Institute. Since 2019, he has been an assistant professor in Department of Power Mechanical Engineering at National Tsing Hua University. His research interests are in brittle material grinding/polishing processes and ultraprecision machining. (weilu@pme.nthu.edu.tw)

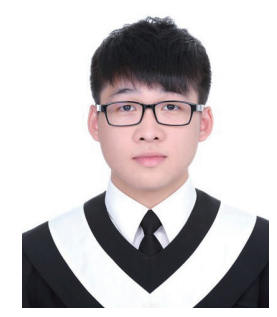

Hong-Chang Chen received his B.S. degree in Department of Mechanical Engineering from National Cheng Kung University, Taiwan, in 2018. His research interests are in brittle material lapping/polishing/finishing and manufacturing process monitoring. (jackson850212@gmail.com)

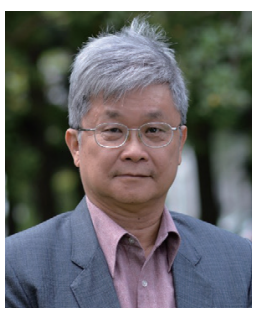

Shih-Chieh Lin received his Ph.D. degree in mechanical engineering from the University of Illinois at Urbana-Champaign, IL, USA. Following his graduation in 1989, he has been with the Department of Power Mechanical Engineering, National Tsing Hua University, Hsinchu, Taiwan. His research interest covers the areas of manufacturing process, modelling and optimization, manufacturing process monitoring, metrology, and microscopy. (sclin@pme.nthu.edu.tw) 